\title{
Arp 116: interacting system or chance alignment? (Research Note)
}

\author{
R. de Grijs` and A. R. I. Robertson
}

\begin{abstract}
Department of Physics \& Astronomy, The University of Sheffield, Hicks Building, Hounsfield Road, Sheffield S3 7RH, UK e-mail: R.deGrijs@sheffield.ac.uk
\end{abstract}

Received 6 July 2006 / Accepted 25 September 2006

\section{ABSTRACT}

\begin{abstract}
Using high spatial resolution Hubble Space Telescope/Advanced Camera for Surveys archival imaging observations of Arp 116, centred on the elliptical galaxy NGC 4649, we explore the novel technique of pixel-by-pixel analysis of the galaxy's colour-magnitude diagram to search for any evidence of recent enhanced star formation due to the apparent tidal interaction with its spiral companion, NGC 4647. From a detailed analysis of the system's geometry, and based on additional circumstantial evidence from extant multiwavelength observations, we conclude that, while there may be grounds for the tidal-interaction assumption for this system, any interaction has thus far been of insufficient strength to trigger an enhanced level of recent star formation in the elliptical component, although close inspection of our colour images shows a faint excess of bluer pixels (a $\sim 0.20$ mag bluer "loop") in the elliptical galaxy on the side of the spiral companion. Given that there appears to be a moderate reservoir of available gas for ongoing star formation (although at low column density), this suggests that we are currently witnessing the onset of the tidal interaction between NGC 4647 and NGC 4649. In addition, the triggering of new star formation in NGC 4649 may be significantly impeded due to the much lower mass of the spiral component.
\end{abstract}

Key words. galaxies: individual: Arp 116 - galaxies: individual: NGC 4647 - galaxies: individual: NGC 4649 galaxies: interactions - galaxies: photometry - galaxies: stellar content

\section{Introduction}

One of the major achievements of the Hubble Space Telescope (HST) to date has been the discovery that the progenitors of globular clusters (GCs), once thought to be the oldest building blocks of galaxies, continue to form until today (e.g., de Grijs et al. 2003b, 2005). HST observations allow us to resolve and study GC systems far beyond the well-studied GC systems in the Milky Way and the Magellanic Clouds.

Young massive star clusters (YMCs; $M_{\mathrm{cl}} \gtrsim 10^{5} M_{\odot}$ ) have been detected in a great variety of actively star-forming galaxies. Systems containing a large number of YMCs are most often interacting spiral-spiral pairs (e.g., the "Antennae" galaxies, Whitmore \& Schweizer 1995; NGC 7252, Whitmore et al. 1993; the "Mice" and "Tadpole" systems, de Grijs et al. 2003d), since these naturally provide large reservoirs of gas for star and cluster formation. In fact, massive star cluster formation is likely the major mode of star formation in such extreme starburst environments (cf. de Grijs et al. 2003d). Therefore, we can use young and intermediate-age massive star clusters as efficient tracers of the recent violent star formation and interaction history of galaxies by determining accurate ages for the individual clusters, even at those ages when morphological interaction features in their host galaxies have already disappeared.

The formation of YMCs and field stars alike is limited by the supply of gas. The colliding gas masses associated with interacting galaxies determine the violence of the star and cluster formation, as well as the overall gas pressure. Hence the number,

^ Also Visiting Professor, National Astronomical Observatories of China, Chinese Academy of Sciences, 20A Datun Road, Chaoyang District, Beijing 100012, PR China. and perhaps also the nature (such as their masses and compactness), of the YMCs, and the ratio of YMC to field star formation in particular, is expected to depend on Hubble type. While observations of gas-rich spiral-spiral mergers are numerous, starformation tracers in mixed-pair mergers have thus far largely been overlooked, although they are undoubtedly of great interest in the context of the parameter space covered by the star cluster and field star formation processes.

One of the most important open questions in this field, and one that we address in this Research Note, is whether the smaller amount of gas available for star formation in early-type galaxies might act as a threshold for star formation in general, and for massive and compact cluster formation in particular, or possibly result in enhanced field star formation (including star formation in small clusters, $M_{\mathrm{cl}} \lesssim 10^{3} M_{\odot}$ ) with respect to the mode of star formation in massive clusters.

The present analysis is of particular current relevance as ever more sensitive observations increasingly reveal neutral and/or molecular hydrogen in elliptical and early-type spiral galaxies (e.g., Wiklind \& Henkel 1989; Cullen et al. 2003, 2006) and in late-stage merger remnants, which might be the progenitors of present-day elliptical galaxies (cf. Georgakakis et al. 2001). Star (cluster) formation in early-type galaxies, long thought to be essentially dust- and gas-free environments, has therefore returned to the forefront of interest.

\section{Object selection, data and data reduction}

\subsection{Arp 116: selection rationale}

We selected Arp 116, a combination of the bright $\left(M_{\mathrm{B}}=\right.$ -21.48 mag) early-type (E2) galaxy NGC 4649 (M 60) and its 
Sc-type companion NGC $4647\left(M_{\mathrm{B}}=-19.81 \mathrm{mag}\right)$, as our prime target. This is one of the closest systems of mixed-type interacting galaxies, at a distance of $\sim 16.8 \mathrm{Mpc}$ in the Virgo cluster (Tonry et al. 2001; based on surface brightness fluctuations (SBF), see Sect. 4.2), and with a projected separation of $\sim 2.5 \equiv 12 \mathrm{kpc}$. At this distance, the resolution of the Advanced Camera for Surveys (ACS)/Wide Field Camera (WFC) on board the HST, $\sim 0.05$ arcsec pixel $^{-1}$ (corresponding to $\sim 4 \mathrm{pc}$ ), allows us to (marginally) resolve and (thanks to their intrinsic brightnesses) robustly identify individual star clusters of "typical” GC size (i.e., with typical half-mass radii of $R_{\mathrm{hm}} \sim 5 \mathrm{pc}$ ).

The Arp 116 system presents an ideal configuration for our case study into the impact of gravitational interactions on the triggering of star formation in the early-type member. First, studies of the nucleus of NGC 4649 by Rocca-Volmerange (1989) show an excess of far-UV emission, which is indicative of a relatively high molecular gas density. She postulates this to be either left over from the main star-formation episode in the galaxy, or the result of accretion over the galaxy's lifetime. In the context of her "UV-hot" model of galaxy evolution, Rocca-Volmerange (1989) interprets the presence of the far-UV excess in NGC 4649 as caused by a mostly continuous star-formation rate, although at a level (independently confirmed by X-ray observations) below her detection threshold, unless the newly-formed stars are all concentrated in a very dense cluster of stars. With our new $H S T$ observations, we can probe significantly fainter and at higher spatial resolution than this earlier work, while our passband coverage is also sensitive to the signatures of recent star formation.

Secondly, Randall et al. (2004) confirm that the X-ray bright elliptical galaxy is characterised by a dominant excess of bright soft X-ray emission, predominantly in its nuclear area, at a temperature of $k T \approx 0.80 \mathrm{keV}$ (and a model-dependent metal abundance within a factor of 2 of solar; but see Randall et al. 2006). They interpret this as thermal emission from interstellar gas, combined with hard emission from unresolved low-mass X-ray binaries. Moreover, Böhringer et al. (2000) report the galaxy to have an extended thermal X-ray halo.

Thirdly, Huchtmeier et al. (1995) and Georgakakis et al. (2001) determined the total HI gas mass in NGC 4649 at, respectively, $M_{\mathrm{HI}}<1.62 \times 10^{8}$ and $<4 \times 10^{8} M_{\odot}$. Contamination of this mass by the gas in the late-type companion is thought to be small. Finally, Cullen et al. (2006), using CO observations, place an upper limit on the molecular gas mass of $M_{\text {molec }}<$ $0.72 \times 10^{7} M_{\odot}$.

The presence of this moderate amount of atomic and molecular gas, combined with the likely disturbance caused by the apparent interaction (possibly linked to enhanced star-formation efficiencies), suggests that dense gas flows could result in principle, possibly followed by their turbulent collapse into stars and possibly (massive) star clusters. In fact, Kundu \& Whitmore (2001; see also Larsen et al. 2001) used archival HST/WFPC2 data of (a section of) NGC 4649, in which they detect a significant number (several tens) of blue clusters - comprising only the bright end of the GC luminosity function. Based on their $[0<(V-I)<0.5]$ mag colours, these clusters could be as young as $20 \mathrm{Myr}$ for solar metallicity, or $\$ 100 \mathrm{Myr}$ if their metallicity is as low as $0.02 Z_{\odot}$.

\subsection{Observations and reduction}

We obtained archival observations of the NGC 4647/9 system, centred on NGC 4649, taken with the ACS/WFC on board HST (GO-9401, PI Côté) through the F475W and F850LP broad-band filters on UT 2003 June 17, with exposure times of 750 and $1120 \mathrm{~s}$, respectively. These filters closely correspond to the Sloan Digitial Sky Survey (SDSS) $b$ and $i$ bands. The ACCUM imaging mode was used to preserve dynamic range and to facilitate the removal of cosmic rays.

For the purpose of the research reported on in this Research Note, we used the standard on-the-fly data reduction pipeline (CALACS) in IRAF/STSDAS ${ }^{1}$, providing us with images corrected for the effects of flat fielding, shutter shading, cosmic ray rejection and dark current. The use of the latest flat fields is expected to result in a generic large-scale photometric uniformity level of the images of $\sim 1 \%$. We used the final, dithered images produced by the most recent release of the PyDrizzle software tool. PyDrizzle also performs a geometric correction on all ACS data, using fourth-order geometric distortion polynomials, and subsequently combines multiple images into a single output image and converts the data to units of count rate at the same time. We registered the individual images obtained for both passbands to high (subpixel) accuracy, using the Iraf IMALIGN routine.

\section{Pixel-by-pixel analysis}

We constructed colour-magnitude diagrams (CMDs) on a pixelby-pixel basis from our ACS observations to study the field star population. This technique proved very powerful in our analysis of the ACS early release observations (ERO) of the "Mice" and the "Tadpole" interacting systems (de Grijs et al. 2003d; see also Eskridge et al. 2003). Since the ACS/WFC is somewhat undersampled by its point-spread function at optical wavelengths, the individual pixels are statistically independent. In our previous work on the ACS ERO data we identified several subpopulations from the CMDs, which were found to originate from spatially well-defined regions within the interacting systems.

We applied the same techniques to the ACS images of the NGC 4647/9 system, in order to search for any evidence of CMD features corresponding to regions of enhanced star formation in NGC 4649. Our painstaking analysis of these high-quality HST-based CMD data proved conclusively that NGC 4649 is essentially composed of a similar stellar population mix throughout the entire body of the galaxy. The only specific CMD features of note correspond to (redder) dusty pockets and to the large population of blue and red GCs (see below).

The galaxy's pixel-CMD does not reveal any significant subpopulation of pixels that could be ascribed to (an enhanced level of) more recent star formation, neither throughout the galaxy as a whole nor in spatially confined regions. For the latter purpose, we carefully scrutinized the pixel-CMD behaviour of the galaxy in its four quadrants defined by the major and minor axes, as well as in smaller wedge-shaped slices, all for galactocentric radii between 20 and 90 arcsec $(\sim 1.6-7.2 \mathrm{kpc})$. These radial constraints were imposed on the one hand to avoid the effects of the bright galactic nucleus, and on the other to avoid spurious pixel-CMD values originating from the spiral companion, NGC 4647.

In Fig. 1 we show the pixel-CMDs for the eight wedgeshaped areas studied independently in the galaxy. The locations

\footnotetext{
1 The Image Reduction and Analysis Facility (IRAF) is distributed by the National Optical Astronomy Observatories, which is operated by the Association of Universities for Research in Astronomy, Inc., under cooperative agreement with the US National Science Foundation. STSDAS, the Space Telescope Science Data Analysis System, contains tasks complementary to the existing IRAF tasks. We used Version 3.5 (March 2006) for the data reduction performed in this paper.
} 

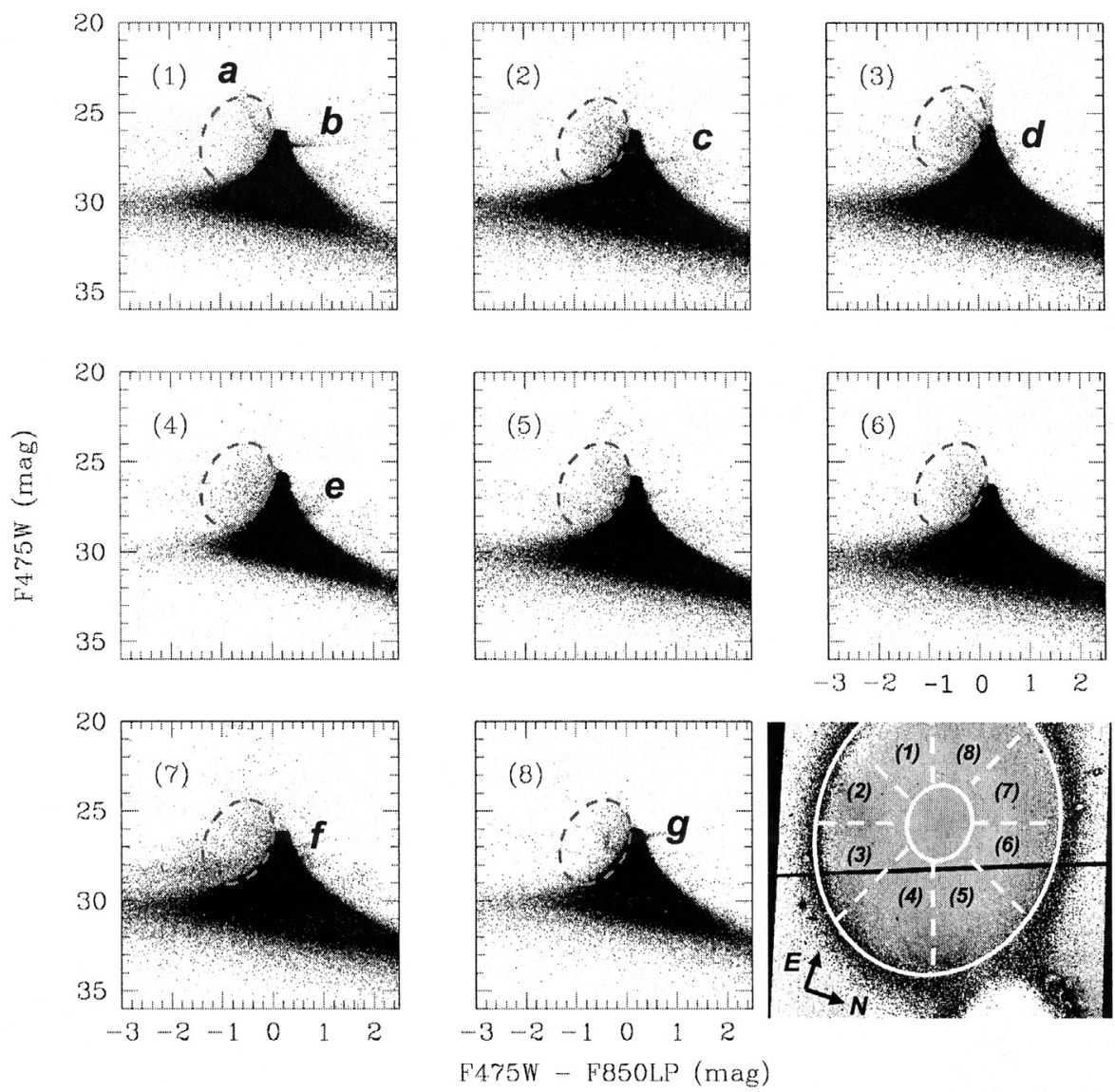

Fig. 1. Pixel-CMDs of eight wedge-shaped fields in NGC 4649. The wedge numbers shown in the top left-hand corner of each panel refer to the wedges indicated on the $(F 475 W-F 850 L P)$ colour image of the galaxy, included in the bottom right-hand corner of the figure. The areas of the "blue excess" pixels are indicated by the dashed ellipses in all panels; the linear spurs and features are identified by labels $a-g$. The colour image shows the full HST/ACS field of view (after geometric corrections), with 4000 ACS pixels, or 200 arcsec, on a side. The colour levels range linearly from $(F 475 W-F 850 L P) \simeq-0.6$ (black) to $\simeq 1.3$ (white).

of which are also indicated here, using a $F 475 W-F 850 L P$ colour image of the field as guidance (the numbers in each panel correspond to the numbered wedges superimposed onto the galaxy image). By applying edge-fitting techniques based on colour histograms at a range of magnitudes we conclude that both the blue and the red edges of the individual wedge pixelCMDs are identical, within the observational uncertainties of $\Delta(F 475 W-F 850 L P) \lesssim 0.08 \mathrm{mag}$, to some extent depending on pixel magnitude (in the sense of smaller uncertainties at brighter magnitudes). For additional comparison purposes, in Table 1 we list the mean $(F 475 W-F 850 L P)$ colours and standard deviations of the bright-peak pixels, for $m_{F 475 W} \leq 27.0$ mag. Since these pixels are unaffected by any recent star formation, the variation in the mean colour reflects the intrinsic colour variation in the main body of the galaxy. We remind the reader that the uncertainties are small because of the large number of data points these statistical measures are based on; they were obtained by slightly varying the magnitude cut-off used to obtain the mean values.

In all cases, and after having taken into account the reddening effect introduced by Poissonian noise (shot noise) statistics at the faintest magnitudes, the mean $(F 475 W-F 850 L P)$ colour was compared to the GALEV simple stellar population (SSP) models (cf. Anders \& Fritze-v. Alvensleben 2003), which include model sets calculated for the full set of HST imaging filters. The mean colour is consistent with old stellar populations, for a range of relevant metallicities (from 0.02 to $1.0 Z_{\odot}$ ).
Table 1. $(F 475 W-F 850 L P)$ colours and standard deviations of the pixels with $m_{F 475 W} \leq 27.0$ mag.

\begin{tabular}{ccc}
\hline \hline Wedge & $\begin{array}{c}\text { Mean } \\
\text { colour (mag) }\end{array}$ & $\begin{array}{c}\text { Standard } \\
\text { deviation }(\mathrm{mag})\end{array}$ \\
\hline 1 & $0.194 \pm 0.004$ & $0.142 \pm 0.012$ \\
2 & $0.188 \pm 0.003$ & $0.098 \pm 0.003$ \\
3 & $0.205 \pm 0.002$ & $0.089 \pm 0.002$ \\
4 & $0.217 \pm 0.001$ & $0.088 \pm 0.003$ \\
5 & $0.205 \pm 0.001$ & $0.122 \pm 0.006$ \\
6 & $0.184 \pm 0.001$ & $0.121 \pm 0.002$ \\
7 & $0.181 \pm 0.002$ & $0.166 \pm 0.010$ \\
8 & $0.194 \pm 0.002$ & $0.100 \pm 0.005$ \\
\hline
\end{tabular}

In Fig. 1 we have also indicated the area where we observe a "blue excess" in all wedges (dashed ellipses), as well as a number of almost linear spurs and feather-like features on either the blue or red side of the pixel-CMD of the main galaxy body $(a-g)$. In all cases, the "blue excess" pixels correspond to a combination of clearly identifiable blue GCs (which appear as spatially clumped blue excess pixels) and shot noise at the faintest isophote covered by our detailed investigation (down to $m_{F 475 W}=28.5 \mathrm{mag}$ ); the latter could also be clearly identified based on its radially random spatial distribution and random noise characteristics. (In wedge 7, the apparently larger amount of these blue excess pixels is due to the presence of a foreground star with similar colours.) We note that there is no clear 
enhancement of blue excess pixels on the side of the galaxy facing its spiral companion; the amount of such pixels is similar or even slightly reduced with respect to the rest of the galaxy.

Similarly, the spurs and feathers $(a-g)$ correspond to the large (red and blue) GC population, as well as to shot noise at the faintest isophote covered by our study. This also applies to the spurs extending to brighter magnitudes starting from the cutoff magnitude of the main body of the galaxy in wedges 3 and 4 . Once again, the nature of these pixels which led us to identify them as GCs or noise is very clear from their spatial distribution and brightness characteristics. We note that these spurs seem to occur predominantly on the side of the galaxy away from its spiral companion, which is consistent with the distribution of the blue excess pixels.

Thus, we conclude that the apparent tidal interaction with NGC 4747 (but see Sect. 5 for a discussion on the robustness of the tidal-interaction assumption) has had a negligible effect (if any) on the recent star-formation activity in NGC 4649. However, close inspection of the $(F 475 W-F 850 L P)$ colour image in the bottom right-hand panel does show a darker (bluer) loop of pixels close to the spiral companion. This may be the first evidence of star formation in the elliptical component induced by the close encounter between these two galaxies. The mean $(F 475 W-F 850 L P)$ colour of this blue loop is $-0.04 \pm$ 0.01 , i.e., approximately 0.20 mag bluer than the pixels in the brightest part of the main body of the galaxy ${ }^{2}$. Since these blue pixels occur at the faint limit of our observations, they cannot be distinguished in the individual pixel-CMDs of wedges 4 and 5 because of the overall increase of the observational uncertainties at low brightnesses. However, because the blue loop appears to be a distinct feature in colour space, at a level unexpected from potential flat-field variations after MULTIDRIZZLE and geometric corrections ( $\ll 1$ per cent; Pavlovsky et al. 2006; see also STScI Analysis Newsletter for ACS, 9 August 2002), we believe this to be a real feature intrinsic to the galaxy's stellar population. We also point out that this colour variation of $\sim 0.20 \mathrm{mag}$ exceeds the intrinsic colour variations seen in Table 1, thus further suggesting that this is indeed a real feature.

\section{Interaction or chance alignment?}

Our search for young bright blue pixels signifying recent star formation, particularly in the south-eastern quadrant of the elliptical, has proven futile. This indicates that there have been no recent significant episodes of massive star formation within NGC 4649, at least not on large spatial scales. This implies that either the Jeans mass criterion for the formation of massive star clusters was not met (or, at least, that the threshold for star formation may not have been met) or that the galaxies may not actually be interacting.

\subsection{Conditions for the onset of star formation}

There are a number of possible reasons as to why the Jeans mass criterion, or the threshold conditions for star formation to proceed, may not have been met in NGC 4649. The most likely of these seem to be that (i) there may be insufficient gas; (ii) the gas density may be too low with respect to the strength of the

\footnotetext{
${ }^{2}$ A back-of-the-envelope calculation suggests that if the colours in Table 1 represent the old stellar population, and that (depending on metallicity) a population reddens by $\sim 1.0$ mag from 10 Myr to $1 \mathrm{Gyr}$, the blue-loop pixels contain approximately a $\$ 30$ per cent contribution from a young, recently formed stellar population.
}

postulated gravitational interaction; or (iii) the temperature may be too high for any collapse to proceed unimpeded. The latter is of concern in view of the X-ray temperature quoted in Sect. 2.1, although this temperature is comfortably in the range expected for star-forming and starburst galaxies (see e.g. Hartwell et al. 2004, for the starburst galaxy NGC 4214).

Regarding options (i) and (ii), from the example of the large interacting spiral galaxy NGC 6745a,b and its much smaller early-type companion NGC $6745 \mathrm{c}$, there is evidence that star and cluster formation in early-type, gas-poor galaxies may be triggered if the gravitational interaction is sufficiently violent (de Grijs et al. 2003a). We will return to the issue as to whether the interaction in the Arp 116 system is sufficiently violent (and whether the galaxies are sufficiently close to one another) in Sects. 4.2 and 5. However, we point out here that Cullen et al. (2006) report an upper limit to the detectable HI column density (at the $3 \sigma$ level) of $N_{\mathrm{HI}} \lesssim(3.5 \pm 0.3) \times 10^{20} \mathrm{~cm}^{-2}$. They emphasize that this is a factor of four lower than that observed in the elliptical galaxy NGC 1410 by Keel (2004), which does not show strong evidence of recent star formation either, based on $H S T /$ STIS imaging and WIYN spectral mapping techniques. In fact, a conservative back-of-the-envelope estimate of the mean surface density of the atomic and molecular gas in NGC 4649, implies values of $\lesssim 0.1 M_{\odot} \mathrm{pc}^{-2}$. We based this estimate on a total gas mass estimate of $2 \times 10^{8} M_{\odot}$ (cf. Sect. 2.1), smoothly distributed within the galaxy's $D_{25}$ isophote $\left(7^{\prime} \cdot 4 \times 66^{\prime} 0\right)$. This is an order of magnitude lower than the (critical) threshold densities for star formation derived by Kennicutt (1989) for the outer regions of spiral galaxies, a situation that is even worsened if we realise that the HI distribution is often distributed well beyond the optical extent of most galaxies.

This suggests that the possibly weak interaction, combined with the low density of the interstellar gas in the galaxy, may not be sufficiently conducive to trigger star-formation rates at the level that can be observed with the current-best instrumentation.

We should also point out that the mass ratio of the NGC 4647/9 system is opposite to that of the NGC 6745 system; from $K$-band imaging, Cullen et al. (2006) conclude that the ratio of the masses of the early with respect to the late-type component is $\sim 5.2$. If a gravitational encounter occurs between unevenly matched galaxies, provided that they contain sufficient gas reservoirs, then - as expected, both intuitively and based on a large number of dynamical simulations - the effects of the gravitational interaction are much more pronounced in the smaller galaxy. For instance, when we compare the impact of the interaction as evidenced by star cluster formation (which we take as the most violent mode of star formation here) between M 82 (de Grijs et al. 2001, 2003b,c) and M 81 (Chandar et al. 2001), the evidence for enhanced cluster formation in the larger galaxy is minimal if at all detectable.

\subsection{The distance to Arp 116}

Despite these galaxies being members of the Virgo cluster (they are, in fact, projected to be close to the cluster core), their distance estimates are rather uncertain. Clearly, for the galaxies to interact, they need to be sufficiently close to one another in order to respond to their mutual gravitational effects.

Therefore, we embarked on a detailed literature search for reliable distance measurements to both galaxies. The most comprehensive review of the distance to NGC 4649, including an extensive bibliography up to July 1999, was published by the HST Key Project on the Extragalactic Distance Scale (Ferrarese et al. 2000a). The secondary distance indicators they 
used include the method of SBF, the bright-end cut-off of the Planetary Nebula Luminosity Function (PNLF), and the peak of the GC Luminosity Function (GCLF); homogeneous calibration relations of these methods, relating them to the Cepheid distance scale, were published by Ferrarese et al. (2000b). Independent analysis of their sample galaxies based on the $D_{n}-\sigma$ method resulted in fully consistent distance measurements (Kelson et al. 2000), thus instilling confidence in the robustness of these distance calibrations. Here we will discuss the additional relevant evidence regarding the distance to NGC 4649 presented in studies following the 1999 review.

Ferrarese et al. (2000a,b) adopted as the best average distance modulus to NGC 4649, $m-M=31.09 \pm 0.08 \mathrm{mag}$. Subsequent measurements largely agree with this value (e.g., Tonry et al. 2001; Di Criscienzo et al. 2006; Marín-Franch \& Aparicio 2006; but see Neilsen \& Tsvetanov 2000). This distance modulus corresponds to a physical distance to NGC 4649 of $D=16.52 \pm 0.60 \mathrm{Mpc}$. For comparison, if we use the galaxy's systemic velocity, $v_{\text {sys }}=1117 \pm 6 \mathrm{~km} \mathrm{~s}^{-1}$ (Ferrarese et al. 2006), and a Hubble constant $H_{0}=67 \mathrm{~km} \mathrm{~s}^{-1} \mathrm{Mpc}^{-1}$, the corresponding distance is in close agreement, at $D_{H_{0}}=16.67 \pm 0.09 \mathrm{Mpc}$ (although we point out that large-scale peculiar motions may affect this result).

In addition, Larsen et al. (2001) used HST/WFPC2 data to obtain the NGC 4649 GCLF based on 345 GCs. They deduced a turn-over magnitude of $m_{V}=23.58 \pm 0.08 \mathrm{mag}$ for the entire GC sample, and $m_{V}=23.46 \pm 0.13$ mag for the GCs in the blue peak, which are usually associated with the oldest GC population in a galaxy making up the "universal" GCLF (e.g., Fritze-v. Alvensleben 2004). Using the Ferrarese et al. (2000b) distance calibration of GCLFs in the Virgo cluster, these turnover magnitudes correspond to distances of $D_{\mathrm{GCLF}}=17.22_{-2.29}^{+2.64}$ and $D_{\mathrm{GCLF}}=16.29 \pm 2.50 \mathrm{Mpc}$, respectively. The latest study by Forbes et al. (2004), using GMOS on Gemini-North, detected 2647 GC candidates; they find a turn-over magnitude of $m_{I}=23.17 \pm 0.15 \mathrm{mag}$. However, as we are specifically interested in the distance to NGC 4649, we are limited to $V$-band measurements for reasons of observational robustness. For this reason we convert their $I$-band turnover magnitude to a $V$-band magnitude, using the GALEV SSP models. We adopt a Salpeter stellar initial mass function covering a mass range from 0.1 to $70 M_{\odot}$ and a metallicity of $0.2 Z_{\odot}$. This yields $(V-I)=1.03$ at an age of $10 \mathrm{Gyr}$, and hence we deduce $m_{V} \simeq 24.2 \mathrm{mag}$. Adopting an absolute turn-over magnitude of $M_{V}=-7.3$ (Harris et al. 1991), gives a distance of $20.0 \mathrm{Mpc}$; using Ferrarese et al.'s (2000b) calibration, we derive a distance of $22.9 \mathrm{Mpc}$ to the galaxy. Considering the manipulation we had to go through in order to reach this result, the uncertainty on these I-band GCLF distances is $\gtrsim 4 \mathrm{Mpc}$.

We base our initial analysis of the distance to the spiral companion, NGC 4647, on the compilation of measurements by Solanes et al. (2002). We also consider the work by Schöniger $\&$ Sofue (1997), who carefully compared the reliability of using the Tully-Fisher relation (TFR) based on both HI and CO observations (locally calibrated using Cepheid distances). All of the Solanes et al. (2002) measurements are TFR based.

Solanes et al.'s (2002) best distance modulus to NGC 4647, based on homogenisation of the available distance measurements, $m-M=31.25 \pm 0.26 \mathrm{mag}$, corresponds to a physical distance of $D=17.78_{-2.00}^{+2.26} \mathrm{Mpc}$. The spread in distance measurements obtained for this galaxy is in essence within $\sim 2 \sigma$ of this value, and straddled by the TFR distances of Schöniger \& Sofue (1997): $D_{\mathrm{CO}}=17.5 \mathrm{Mpc}$ and $D_{\mathrm{HI}}=22.0 \mathrm{Mpc}$. For comparison, the galaxy's recessional velocity of $v_{\mathrm{sys}}=1419 \pm 63 \mathrm{~km} \mathrm{~s}^{-1}$ (from the compilation of Falco et al. 1999; but note that the optical and HI $21-\mathrm{cm}$ velocities differ by $\sim 30 \mathrm{~km} \mathrm{~s}^{-1}$; cf. Huchtmeier \& Richtler 1986) implies a distance of $D_{H_{0}} \simeq 20.6 \mathrm{Mpc}$ (or $18.9 \mathrm{Mpc}$ if we base our estimate on the recessional velocity of $v_{\mathrm{LG}} \simeq 1305 \mathrm{~km} \mathrm{~s}^{-1}$ with respect to the barycentre of the Local Group; Helou et al. 1984).

Thus, here we conclude that the two galaxies are most likely within 1-1.5 Mpc of each other. Their velocity differential of order $300 \mathrm{~km} \mathrm{~s}^{-1}$ (e.g., Gavazzi et al. 1999; Cullen et al. 2006) is also well within the velocity dispersion of the Virgo cluster as a whole, $\sigma_{\text {Virgo }} \sim 821 \mathrm{~km} \mathrm{~s}^{-1}$ (e.g., Sandage \& Tammann 1976). It is, however, unclear whether the galaxies are approaching or receding from each other; even very careful modelling of their luminosity profiles in search of extinction features at large radii (White et al. 2000) proved inconclusive as to which galaxy might be in front of the other.

\section{The final verdict?}

Owing to the uncertainties in the absolute calibration of the various secondary distance indicators we needed to rely on in Sect. 4.2, combined with the relatively low gas content in the elliptical galaxy, we can neither rule out nor confirm that both galaxies are in fact sufficiently close to expect signs of gravitational disturbances ${ }^{3}$.

Rubin et al. (1999) point out that, although their long-slit optical spectra show that the kinematics of the spiral component, NGC 4647, appear nearly normal (but see Young et al. 2006), the $\mathrm{H} \alpha$ and molecular $\left(\mathrm{CO}\right.$ and $\left.\mathrm{H}_{2}\right)$ gas disks are clearly asymmetric (Koopmann et al. 2001; Young et al. 2006), while the more extended HI disk is also slightly extended toward NGC 4649 (Cayatte et al. 1990), but not nearly by as much as the molecular gas (Young et al. 2006). This might reflect an HI infall scenario, as Cayatte et al. (1990) suggest, or possibly the onset of a tidal interaction between the two galaxies (Rubin et al. 1999; Cullen et al. 2006). The latter idea is supported by results from observations as well as numerical simulations that the most apparent effects of ongoing tidal encounters tend to occur after the period of closest approach (e.g., Moore et al. 1998; Rubin et al. 1999; Young et al. 2006). However, from their detailed analysis of the gas pressure across the disk of NGC 4647, Young et al. (2006) conclude that ram pressure effects alone, postulated to be caused by tidal forces from NGC 4649, do not explain the observations entirely satisfactorily. Instead, they favour a lopsided gravitational potential as the primary cause for the asymmetries observed, akin to those commonly seen in spiral galaxies.

Bender et al. (1994) analysed the resolved optical kinematic profiles of NGC 4649 along both its major and minor axes, out to radii of $\sim 40$ arcsec. Interestingly, they found evidence for a weak asymmetry in the line-of-sight velocity dispersion (LOSVD), as well as negative $h_{3}$ values (i.e., third-order Gauss-Hermite coefficients, giving higher-order kinematic information about the deviations of the LOSVD from a Gaussian distribution), along the galaxy's major axis (roughly pointing toward the spiral companion). On the other hand, the minor-axis kinematics do not show any significant asymmetries. Negative $h_{3}$ values are normally seen in disky elliptical galaxies, and in ellipticals with kinematically decoupled cores, but Bender et al. (1994) did not find any photometric or kinematic evidence for the presence of such components. In addition, they concluded that the observed

\footnotetext{
3 In fact, we realise that the Milky Way and M 31 are also well inside the uncertainty range derived in Sect. 4.2, yet show no sign of any mutual interaction...
} 
asymmetry was unlikely caused by projection effects. These conclusions are corroborated by both De Bruyne et al. (2001) and Pinkney et al. (2003), who traced the galaxy's kinematics out to $\sim 90$ and 40 arcsec, respectively, along its major axis. De Bruyne et al. (2001), in particular, detected a $\sim 70 \mathrm{~km} \mathrm{~s}^{-1}$ difference between the rotation curves on either side of the galactic centre at their outermost measured radii, just beyond the galaxy's effective radius. These results provide circumstantial support for the tidal interaction idea, with NGC 4647 thought to have caused this minor disturbance of the NGC 4649 kinematics.

Thus, while there may be grounds for the tidal-interaction assumption in the case of the Arp 116 system, any interaction has thus far been of insufficient strength to trigger an enhanced level of recent star formation in the elliptical galaxy component (with the possible exception of a $\sim 0.20$ mag bluer "loop" of pixels in the elliptical galaxy on the side of its spiral companion). This was shown conclusively by the null result we obtained from our careful analysis of the elliptical galaxy's CMD on a pixel-bypixel basis. This suggests that we are currently witnessing the onset of the tidal interaction between NGC 4647 and NGC 4649. In addition, the threshold for new star formation in NGC 4649 may be a further unmet constraint due to the much lower mass of the spiral component. Detailed numerical ( $N$-body) and hydrodynamical (SPH) modelling, taking into account both the current shape of the gaseous morphology of NGC 4647 (as well as entertaining the possibility of the presence of a lopsided gravitational potential), and the kinematic disturbances seen along the NGC 4649 major axis, are required to shed light on the future evolution of this intriguing system.

Acknowledgements. We acknowledge stimulating discussions with Simon Goodwin and Peter Anders. R.d.G. acknowledges an International Outgoing Short Visit grant to the National Astronomical Observatories of China in Beijing from the Royal Society, and hospitality and support from Profs. X. Q. Na, and L. C. Deng, Dr. J. Na, and Z. M. Jin during the final stages of this project. We are grateful to the referee for suggesting improvements to make this paper more robust. This paper is based on archival observations with the NASA/ESA Hubble Space Telescope, obtained from the ST-ECF archive facility. We acknowledge the use of the HyperLeda database (http://leda.univ-lyon1.fr). This research has also made use of NASA's Astrophysics Data System Abstract Service.

\section{References}

Anders, P., \& Fritze-v. Alvensleben, U. 2003, A\&A, 401, 1063 Bender, R., Saglia, R. P., \& Gerhard, O. E. 1994, MNRAS, 269, 785 Böhringer, H., Voges, W., Huchra, J. P., et al. 2000, ApJS, 129, 435

Cayatte, V., van Gorkom, J. H., Balkowski, C., \& Kotanyi, C. 1990, AJ, 100, 604 Chandar, R., Ford, H. C., \& Tsvetanov, Z. 2001, AJ, 122, 1330
Cullen, H., Alexander, P., \& Clemens, M. 2003, Ap\&SS, 284, 503 Cullen, H., Alexander, P., \& Clemens, M. 2006, MNRAS, 366, 49 De Bruyne, V., Dejonghe, H., Pizzella, A., Bernardi, M., \& Zeilinger, W. W. 2001, ApJ, 546, 903

de Grijs, R., O'Connell, R. W., \& Gallagher, J. S. 2001, AJ, 121, 768 de Grijs, R., Anders, P., Lynds, R., et al. 2003a, MNRAS, 343, 1285 de Grijs, R., Bastian, N., \& Lamers, H. J. G. L. M. 2003b, ApJ, 583, L17 de Grijs, R., Bastian, N., \& Lamers, H. J. G. L. M. 2003c, MNRAS, 340, 197 de Grijs, R., Lee, J. T., Mora Herrera, M. C., et al. 2003d, NewA, 8, 155 de Grijs, R., Parmentier, G., \& Lamers, H. J. G. L. M. 2005, MNRAS, 364, 1054 Di Criscienzo, M., Caputo, F., Marconi, M., \& Musella, I. 2006, MNRAS, 365, 1357

Eskridge, P. B., Frogel, J. A., Taylor, V. A., et al. 2003, ApJ, 586, 923

Falco, E. E., Kurtz, M. J., Geller, M. J., et al. 1999, PASP, 111, 438

Ferrarese, L., Mould, J. R., Kennicutt, R. C., Jr., et al. 2000a, ApJ, 529, 745

Ferrarese, L., Ford, H. C., Huchra, J. P., et al. 2000b, ApJS, 128, 431

Ferrarese, L., Côté, P., Jordán, A., et al. 2006, ApJS, 164, 334

Forbes, D. A., Faifer, F. R., Forte, J. C., et al. 2004, MNRAS, 355, 608

Fritze-v. Alvensleben, U. 2004, A\&A, 414, 515

Gavazzi, G., Boselli, A., Scodeggio, M., Pierini, D., \& Belsole, E. 1999, MNRAS, 304, 595

Georgakakis, A., Hopkins, A. M., Caulton, A., et al. 2001, MNRAS, 326, 1431

Harris, W. E., Allwright, J. W. B., Pritchet, C. J., \& van den Bergh, S. 1991, ApJ, 76,115

Hartwell, J. M., Stevens, I. R., Strickland, D. K., Heckman, T. M., \& Summers, L. K. 2004, MNRAS, 348, 406

Helou, G., Hoffman, G. L., \& Salpeter, E. E. 1984, ApJS, 55, 433

Huchtmeier, W. K., \& Richtler, T. 1986, A\&AS, 64, 111

Huchtmeier, W. K., Sage, L. J., \& Henkel, C. 1995, A\&A, 300, 675

Keel, W. C. 2004, AJ, 127, 1325

Kelson, D. D., Illingworth, G. D., Tonry, J. L., et al. 2000, ApJ, 529, 768

Kennicutt, R. C., Jr. 1989, ApJ, 344, 685

Koopmann, R. A., Kenney, J. D. P., \& Young, J. 2001, ApJS, 135, 125

Kundu, A., \& Whitmore, B. C. 2001, AJ, 121, 2950

Larsen, S. S., Brodie, J. P., Huchra, J. P., Forbes, D. A., \& Grillmair, C. J. 2001, AJ, 121, 2974

Marín-Franch, A., \& Aparicio, A. 2006, A\&A, 450, 979

Moore, B., Katz, N., \& Lake, G. 1998, ApJ, 495, 139

Neilsen, E. H., Jr., \& Tsvetanov, Z. I. 2000, ApJ, 536, 255

Pavlovsky, C., Koekemoer, A., Mack, J., Karakla, D., \& Rose, S. 2006, ACS Data Handbook, Version 5.0, Baltimore: STScI

Pinkney, J., Gebhardt, K., Bender, R., et al. 2003, ApJ, 596, 903

Randall, S. W., Sarazin, C. L., \& Irwin, J. A. 2004, ApJ, 600, 729

Randall, S. W., Sarazin, C. L., \& Irwin, J. A. 2006, ApJ, 636, 200

Rocca-Volmerange, B. 1989, MNRAS, 236, 47

Rubin, V. C., Waterman, A. H., \& Kenney, J. D. P. 1999, AJ, 118, 236

Sandage, A. R., \& Tammann, G. A. 1976, ApJ, 207, L1

Schöniger, F., \& Sofue, Y. 1997, A\&A, 323, 14

Solanes, J. M., Sanchis, T., Salvador-Solé, E., Giovanelli, R., \& Haynes, M. P. 2002, AJ, 124, 2440

Tonry, J. L., Dressler, A., Blakeslee, J. P., et al. 2001, ApJ, 296, 447

White, R. E., III, Keel, W. C., \& Conselice, C. J. 2000, ApJ, 542, 761

Whitmore, B. C., \& Schweizer, F. 1995, AJ, 109, 960

Whitmore, B. C., Schweizer, F., Leitherer, C., Borne, K., \& Robert, C. 1993, AJ, 106,135

Wiklind, T., \& Henkel, C. 1989, A\&A, 225, 1

Young, L. M., Rosolowsky, E., van Gorkom, J. H., \& Lamb, S. A. 2006, ApJ, in press [arXiv:astro-ph/0606410] 